\title{
Brain activity during landmark and line bisection tasks
}

\author{
Metehan Çiçek ${ }^{*}$, Leon Y. Deouell ${ }^{2}$ and Robert T. Knight ${ }^{3}$ \\ Department of Physiology, University of Ankara, Turkiye \\ 2 Department of Psychology, The Hebrew University of Jerusalem, Israel \\ ${ }^{3}$ Department of Psychology and the Helen Wills Neuroscience Institute, University of California, USA
}

\section{Edited by:}

Anna C. Nobre, University of Oxford,

UK

\section{Reviewed by:}

Rik R. Vandenberghe, University

Hospitals Leuven, Belgium; Katholieke Universiteit Leuven, Belgium

Anna C. Nobre, University of Oxford, UK

\section{*Correspondence:}

Metehan Çiçek, Ankara Universitesi Tip Fakultesi Fizyoloji ABD, Dekanlik Binasi, Sihhiye, Ankara TR-06100, Turkiye.

e-mail: cicek@medicine.ankara.edu.tr
Neglect patients bisect lines far rightward of center whereas normal subjects typically bisect lines with a slight leftward bias supporting a right hemisphere bias for attention allocation. We used $\mathrm{fMRI}$ to assess the brain regions related to this function in normals, using two complementary tasks. In the Landmark task subjects were required to judge whether or not a presented line was bisected correctly. During the line bisection task, subjects moved a cursor and indicated when it reached the center of the line. The conjunction of BOLD activity for both tasks showed right lateralized intra-parietal sulcus and lateral peristriate cortex activity. The results provide evidence that predominantly right hemisphere lateralized processes are engaged in normal subjects during tasks that are failed in patients with unilateral neglect and highlight the importance of a right fronto-parietal network in attention allocation.

Keywords: spatial attention, line bisection, fMRI, laterality

\section{INTRODUCTION}

\section{VISUOSPATIAL ATTENTION}

Different models are posited to explain the neural mechanisms of spatial attention. Mesulam $(1981,1990)$ proposed a network model of attention in which posterior parietal cortex, the cingulate cortex and the frontal cortex centered around frontal eye field (FEF) interact to support attention allocation. The cortical components within this network are also connected with subcortical structures including superior colliculus, pulvinar and striatum, which have also been implicated in attention allocation. All these regions are modulated by input from the reticular activating system. According to this model the parietal component provides an internal perceptual map of the external world whereas the frontal component coordinates the motor program for exploration, scanning, reaching and fixating.

Posner and colleagues (Posner, 1995; Posner and Petersen, 1990) modeled attention as consisting of posterior and anterior attention systems, as well as vigilance networks. A posterior network involving the parietal cortex, pulvinar and the superior colliculus was proposed to be involved in performing operations needed to bring attention to a location in space. An anterior network involved anterior cingulate and supplementary motor cortex and was suggested to be activated during detection of salient events and the preparation of appropriate responses. Executive control over voluntary behavior and thought processes are also proposed to be dependent on the anterior network. Several neuroimaging studies using PET or fMRI during visual attention tasks have reported activations in the attention networks supporting Mesulam's and to some degree Posner's models (Corbetta et al., 2000; Gitelman et al., 1999; Hopfinger et al., 2000; Nobre et al., 1997; Vandenberghe et al., 2000). However, the lateralization of the reported activations was not consistent especially for the intra-parietal sulcus (IPS). While some studies report right lateralized activations (Gitelman et al., 1999; Nobre et al., 1997) most report bilateral IPS activations (Corbetta et al., 2000; Hopfinger et al., 2000; Vandenberghe et al., 2000).
Corbetta et al. $(2000,2002)$ reported event related fMRI results related to visuospatial processing and suggested that two brain systems interact to mediate attention. A "dorsal" fronto-parietal network, consisting of the IPS and superior frontal cortex (corresponding to $\mathrm{FEF}$ ), was proposed to be bilaterally activated during voluntary ("top-down") allocation of attention and directing visuomotor behavior. Temporo-parietal junction (TPJ) and inferior frontal regions formed a separate "ventral" network proposed to be activated when salient perceptual events cause re-orientation of attention in a bottom-up manner. According to this model, whereas the dorsal attentional system is bilateral, with some regions responding more strongly when attention is shifted contralaterally, the ventral attentional system is right lateralized, for both left and right shifts of attention (Corbetta and Shulman, 2002; Corbetta et al., 2002). In this study, we addressed this proposed pattern of hemispheric asymmetry using a task different from the one on which Corbetta et al. based their model. The tasks we chose, line bisection and the landmark test, are ones commonly used for testing the attentional deficits of patients with unilateral neglect, a syndrome linked to damage to attentional systems, and in particular the right-lateralized ventral attentional system (Corbetta and Shulman, 2002; Mort et al., 2003).

\section{UNILATERAL SPATIAL NEGLECT}

Unilateral spatial neglect is a frequent outcome of mainly right hemisphere damage. Hemi-spatial neglect is very detrimental to the patients' rehabilitation prognosis due to the prominent attention difficulties and lack of awareness of their deficits (Katz et al., 1999). Unilateral neglect is more frequently associated with right hemisphere lesions and is also more severe subsequent to right rather than left hemisphere lesions (Heilman, 1998). Cortical damage in the right inferior parietal, right temporo-parietal junction, superior temporal, frontal, and cingulate regions as well as certain subcortical structure lesions including the pulvinar and basal 
ganglia may cause neglect (Karnath et al., 2002, 2004; Mort et al., 2003; Vallar, 2001).

In the brunt of these cases both voluntary and involuntary attention is compromised. This could be due the fact each area is involved in both forms of attention or due to transient diaschisis in highly interconnected yet specialized cortical areas as has been reported in PET and fMRI studies (Fiorelli et al., 1991; He et al., 2007; Sobesky et al., 2005).

\section{LINE BISECTION-LANDMARK TASKS AND PSEUDONEGLECT}

Several formal tests have been used to assess unilateral neglect, including search tasks, copying and drawing (Parton et al., 2004). One of the most popular tests is the line bisection, in which patients are required to place a mark in the exact midpoint of a line drawn on a sheet of paper in front of the patient. Neglect patients bisect lines to the right of the true midline, seemingly neglecting part of or underestimating the length of the left side of the line. In contrast, neurologically intact right handed subjects tend to systematically err to the left of center, a phenomenon Bowers and Heilman first referred to as pseudoneglect (Bowers and Heilman, 1980; Bradshaw et al., 1986; Nalçacı et al., 1997; Scarisbrick et al., 1987). The misbisection to the left is not as pronounced as the rightward bias observed in neglect patients. Pseudoneglect was reported to have considerable between subject variation (Manning et al., 1990). However, when assessed with a forced choice tachistoscopic test healthy observers showed consistent bisection errors (McCourt, 2001). The leftward bias in controls is presumed to reflect a right hemisphere attention bias to the left field.

The Landmark task, which requires subjects to judge whether pre-marked lines are correctly bisected, is also used in unilateral spatial neglect studies, and neglect patients typically fail this test as well (Marshall and Halligan, 1995). In contrast to line bisection, the landmark task has a reduced motor load and seems to require limited eye movement (Fink et al., 2000b). This presumably allows one to partially dissociate perceptual from motor aspects of the bisection errors. The fact that subjects have limited motor demands has made the landmark task a test of choice in fMRI studies of spatial attention (Fink et al., 2000a; McCourt and Olafson, 1997). Fink et al. (2000a,b, 2001, 2002) used the Landmark task in a series of fMRI studies and reported superior posterior parietal cortex (or IPS) and temporo-parietal junction (inferior parietal lobule) activations. Activations in both of these locations tended to be more robust on the right hemisphere (Fink et al., 2000a,b, 2001). Assuming that the landmark test requires voluntary, sustained, top-down attention rather then involuntary bottom-up attention, this seems to be at odds with the predictions of the recent model of Corbetta and colleagues, both in involvement of the temporo-parietal junction, part of the putative bottom-up ventral attention system, and in the right lateralization of the IPS activation. However, the descriptions of laterality patterns in specific sub-regions of the posterior parietal cortex in Fink et al.'s work do not allow a clear comparison to the model suggested by Corbetta and Shulman (2002).

We used both Line Bisection and Landmark paradigms in the same fMRI experiment. Both the Line Bisection and the Landmark paradigms require voluntary attention. We reasoned that the neural regions common to both tasks would define key elements of the voluntary attention network, and aimed to examine the pattern of laterality of this activity.

\section{MATERIALS AND METHODS SUBJECTS}

Sixteen right handed subjects with no history of neurological or psychiatric disease volunteered for the study. Due to head movements and other technical problems 11 participants' data were included in the analysis. Subjects gave informed consent according to procedures approved by the University of California Committee for the Protection of Human Subjects.

\section{PROCEDURE}

Functional images were collected during four conditions detailed below. In all conditions basic stimuli were black lines spanning $6.3^{\circ}$ to $8.6^{\circ}$ visual angle (varying randomly stepwise between 11 and $15 \mathrm{~cm}$ width) presented on a white background on a $13.8^{\circ} \times 10.3^{\circ}$ screen $(24 \times 14 \mathrm{~cm})$. Subjects viewed the screen from a mirror mounted on the head coil $10 \mathrm{~cm}$ away from their eyes. The distance between the mirror and the screen was approximately $90 \mathrm{~cm}$. The lines were presented with their right or left edge $4 \mathrm{~cm}$ away from the right or left edge of the screen respectively. This ensured that the lines' midline was never centered horizontally on the screen.

The experimental paradigm (Figure 1) was created and delivered by the Presentation (version 0.55 ) program (Neurobehavioral

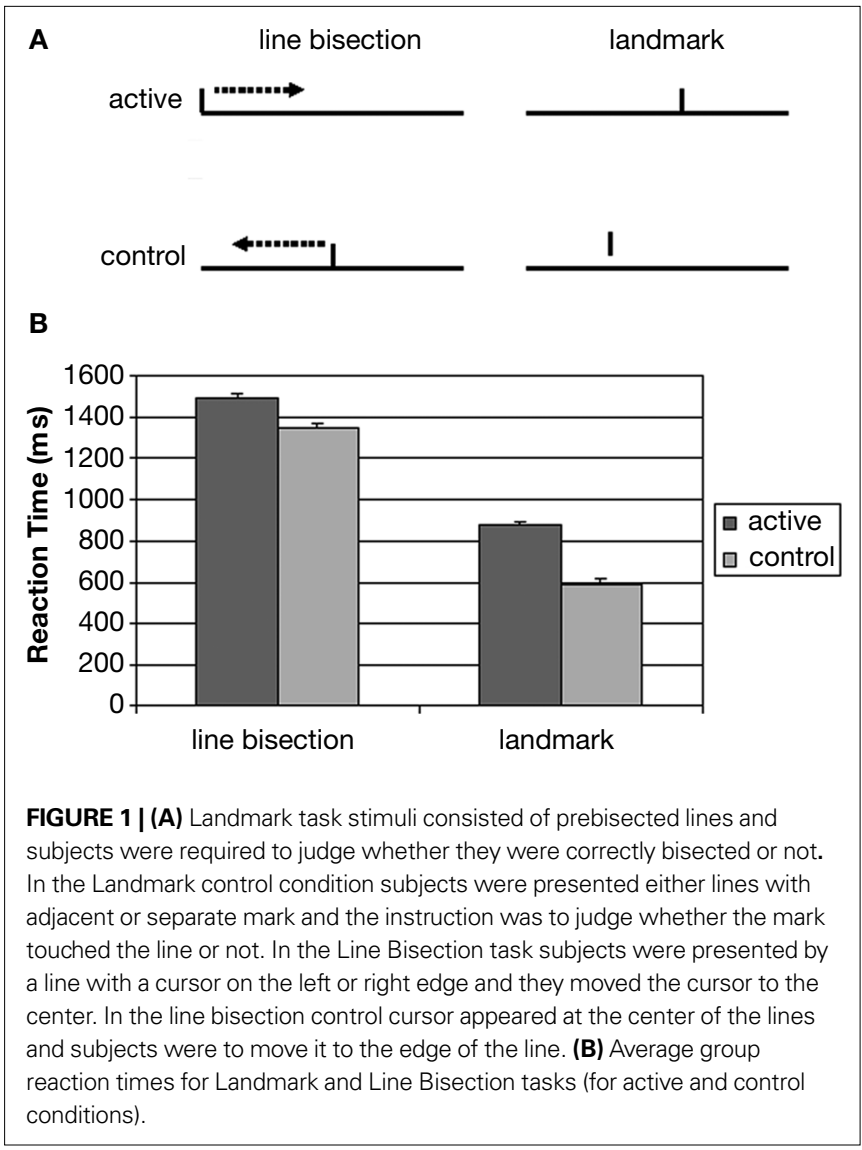


system, CA, USA). Subjects used their right hand for all the responses. Conditions were as follows:

1. Line bisection task (LB): In this paradigm subjects moved a cursor on the presented lines by pressing the left button on a response box for moving the cursor to the left and the right button for moving the cursor to the right. Lines were presented initially with the cursor on the left or right edge of the line ( $50 \%$ probability each). Subjects were instructed to move the cursor to the line's midline, and once there, to confirm by pressing another button at the center of the response box. Bisection error was calculated by averaging absolute deviations from the midline. Bias parameter was computed as a negative score defining a left deviation from the true midline and a positive score for right deviation.

2. Line Bisection control (LBC): Lines were presented with the cursor initially in the midline position. Subjects were required to move the cursor to the left or right edge of the line by pressing one button for left movement and another for right movement as in the Line Bisection task, and once at the edge, to confirm by pressing the third button. Left and right movements were required an equal number of times.

3. Landmark task (LM): The presented lines were prebisected by a short vertical line. Subjects were asked to judge whether the presented lines were prebisected correctly. They pushed one button for correct and another for incorrect response. Bisection marks were placed at the true midline $(p=0.4)$, or deviated to the right or left of the midline by $2.5 \%, 5 \%$ or $7.5 \%$ of the line's length, each deviation presented with a 0.1 probability. Percentage of correct responses was calculated as subjects performance score.

4. Landmark control (LMC): All the stimuli were identical to the Landmark task except that the bisection mark was immediately adjacent to the line as in the Landmark test in $40 \%$ of trials, but was placed slightly above the line and did not actually make contact with it in the remaining $60 \%$ of the trials. Participants were instructed to press one button if the mark made contact with the line, and another button if it was separate from the line. Whether there was a gap between the mark and the line or not was orthogonal to whether the mark correctly bisected the line or not.

5. Low level baseline: In this rest condition participants looked at the white screen without any additional task. The control conditions shared with the experimental conditions the visual display of a horizontal line and a small marker, the motor demands, as well as the requirement to attend to the display. The critical difference between the experimental conditions (LB and LM) and their respective controls is in the fact that the experimental conditions exclusively required a spatial judgment (equality of length of the two parts of the line) which we reasoned requires voluntarily deploying or shifting spatial attention over both sides of space.

The experiment was conducted in a block design. Each block lasted $20 \mathrm{~s}$, including 10 line presentations, each lasting $2 \mathrm{~s}$. Each block was preceded by an instruction screen for $4 \mathrm{~s}$, indicating which of the five conditions will follow. Each fMRI session consisted of three pseudo-randomized repetitions of five conditions and each subject performed 6 runs.

\section{EYE MOVEMENT RECORDING AND ANALYSIS}

Tasks were performed without eye fixation, as in the clinical tests after which the tasks are designed. Eye position was monitored in the scanner at $60 \mathrm{~Hz}$ with an infrared video graphic camera equipped with a telephoto lens (model 504LRO; Applied Science Laboratories, Bedford, MA, USA) that focused on the right eye viewed from a small dielectric flat surface mirror mounted inside the RF coil. Nine-point calibrations were performed at the beginning of the session and between runs when necessary. Eight subjects' eye data were available out of 11 because of technical difficulties. The eye movement data were analyzed using ILAB software (Gitelman, 2002). For each of the four conditions (not for resting condition), we calculated the length of scan path of the subject's gaze on the screen.

\section{fMRI PROCEDURE}

Imaging was performed using a 4.0-Tesla Varian Inova scanner equipped with a fast gradient system for echoplanar imaging and a standard RF head coil. A gradient-echo, echo-planar sequence $(\mathrm{TR}=2000 \mathrm{~ms}, \mathrm{TE}=28 \mathrm{~ms}$, matrix size $=64 \times 64, \mathrm{FOV}=22.4 \mathrm{~cm})$ was used to acquire data sensitive to the blood oxygen level dependent (BOLD) signal. Each functional volume contained eighteen $6 \mathrm{~mm}$ thick axial slices with $1 \mathrm{~mm}$ gap, which covered the subject's whole brain from the vertex to the base of cerebellum. Using a midsagital scout image, the slices were oriented parallel to the anterior-posterior commissure (AC-PC). Each subject was scanned in 6 runs of 180 volumes $(3$ repetitions $\times 5$ conditions $\times 12$ TRs per condition, including the instructions).

\section{fMRI DATA ANALYSIS}

Data processing and analysis were performed using MATLAB (The Mathworks, Inc., Natick, MA, USA) and SPM5 (Statistical Parametric Mapping software; Wellcome Department of Cognitive Neurology, London, UK) ${ }^{1}$. SPM was used for realignment, spatial normalization, smoothing and creating statistical maps of significant relative regional BOLD response changes (Friston et al., 1995b,c).

The first 10 images of each time series, during which a steady state tissue magnetization was achieved, were discarded. The remaining 180 volumes were realigned to correct for head movements between scans. The functional images were then coregistered to the high resolution $3 \mathrm{D}$ anatomical image. Structural and functional images were spatially normalized into a standardized anatomical framework using the default T1 template provided in SPM5, based on the averaged-brain of the Montreal Neurological Institute and approximating the normalized probabilistic spatial reference frame of Talairach and Tournoux (1988). Smoothing was performed with a Gaussian kernel of $10 \mathrm{~mm}$ full-width half-maximal.

Low-frequency cosine waves modeled and removed subjectspecific low frequency drifts in signal, and global means were normalized by proportional scaling. Data were analyzed using the general linear model as implemented in SPM5, modeling the conditions using boxcar functions convolved with a "canonical" hemodynamic response function (Friston et al., 1995a).

Statistical parametric maps for specific effects were calculated by applying appropriate contrasts to the parameter estimates. We

${ }^{1}$ http://www.fil.ion.ucl.ac.uk 
obtained one map per subject per contrast, and put them into one-sample $t$-tests random-effect analyses, allowing inferences to the general population. The presented results are significant at a level of at least $p<0.05$ corrected for multiple comparisons. Voxels presenting a $p<0.001$ and belonging to clusters of at least 41 voxels were considered as activated. These parameters were chosen on the basis of Monte Carlo simulations processed with the AlphaSim program ${ }^{2}$. Common activities for Landmark and Line Bisection tasks were assessed. To this end LM-LMC contrast was inclusively masked by LB-LBC (threshold for masking image was $p<0.05$ uncorrected). The differential activations between the Landmark and the Line Bisection was also obtained with [(LM-LMC) $-($ LB-LBC) $]$ and [(LB-LBC) - (LM-LMC)] contrasts. Masking was applied to the interaction contrasts to exclude the effect of deactivations. [LM-LMC] - [LB-LBC] was exclusively masked with LBC-LB and [LB-LBC] - [LM-LMC] was exclusively masked with LMC-LM.

A region of interest (ROI) analysis was performed based on the activated clusters resulting from the Landmark and Line bisection conjunction analysis. To reveal the significance of the lateralization in the results symmetrical left hemisphere ROI was created by mirroring the right hemisphere cluster resulting from the conjunction analysis. That is, following normalization, the sign of the $\mathrm{x}$ coordinates of the active cluster found over the right hemisphere was flipped to determine the mirror region in the left hemisphere. Average BOLD responses were extracted from and averaged within these ROIs from the individual subject images. Wilcoxon Signed Rank Test was used to compare the hemodynamic responses of the left and right ROIs.

\section{RESULTS \\ BEHAVIORAL AND EYE MOVEMENT DATA}

The Landmark task performance (percent correct) of subjects was high $($ mean $=78 \%$, S.E. $\pm 1.7 \%)$. Line bisection scores were as follows: Bisection error, mean $=11.9 \%$, S.E. $\pm 1.1 \%$; Bias, mean $=-0.1$, S.E. \pm 0.7 .

A two-way ANOVA was performed to investigate the effects of task (Landmark or Line Bisection) and condition (active or control) upon reaction times (RT) (see Figure 1 lower panel). The subjects were faster during the Landmark compared to the Line Bisection task [main effect of Task: $F(1,10)=1276, p<0.001$ ] The responses were also faster during control conditions compared to the active task conditions [main effect of condition: $F$ $(1,10)=182, p<0.001]$. These two effects significantly interacted however, as the active versus control RT difference was larger in the Landmark than in the Line Bisection task $[F(1,10)=30$, $p<0.001]$.

A similar two-way ANOVA was performed to investigate the eye movement data. The effects of task (Landmark or Line Bisection) and condition (active or control) upon the average scan path was assessed. The average scan path (in pixels) was longer during Line Bisection compared to the Landmark [main effect of Task: $F(1,7)=9.9, p<0.05]$. The main effect of condition was not significant $(p>0.05)$. Task and condition effects significantly interacted $[F(1,7)=15.5, p<0.01]$. While there was no eye movement difference $(p>0.05)$ between Landmark task

${ }^{2}$ http://afni.nimh.nih.gov/pub/dist/doc/manual/AlphaSim.pdf
$(3175 \pm 478$; mean \pm S.E. $)$ and Line Bisection tasks (3249 \pm 553$)$, the average scan path was longer $\left(t_{1,7}=4.8, p<0.01\right)$ in the Line Bisection control $(4005 \pm 516)$ than in the Landmark control $(1846 \pm 424)$.

\section{fMRI DATA}

\section{Landmark task}

The Landmark task (LM-LMC contrast) activated right IPS, anterior cingulate girus, and right lateral peristriate cortex $(p<0.05$ corrected for multiple comparisons, see Table 1 and Figure 2A).

\section{Line bisection task}

The Line bisection task (LB-LBC contrast) activated right IPS, right FEF and right lateral peristriate cortex $(p<0.05$ corrected for multiple comparisons, see Table 1 and Figure 2B).

\section{Common activities for Landmark and Line Bisection}

The activity related to the two tasks (compared to their respective controls) overlapped in right hemisphere IPS and LPS (see Table 2 and Figure 3). Since no left IPS activity was found, ROI analysis was performed based on right IPS and its left hemisphere mirror site (see "Materials and Methods"). Right IPS activity (percentage signal change) was greater than left for both LM-LMC $\left(z_{1,10}=2.9\right.$, $p<0.01)$ and LB-LBC $\left(z_{1,10}=2.3, p<0.05\right)$ contrasts. While this left-right comparison may seem biased by the fact that the left ROI was created based on the right IPS ROI which was defined functionally, it is only meant to reaffirm the difference between the two hemispheres in this region. Note that simply showing that the right IPS shows activity whereas the left does not is insufficient statistically to establish this difference. In an attempt to define the left ROI functionally we reduced the threshold to 0.05 , uncorrected. This revealed a cluster of 2 voxels at $[-22,-60,50]$ which were already included in the mirror ROI described above. Thus, although the selection of the left IPS ROI was based on the right IPS ROI, the left IPS ROI did reflect the maximal activity for this contrast over the left hemisphere.

\begin{tabular}{|c|c|c|c|c|c|c|}
\hline $\begin{array}{l}\text { Brain } \\
\text { area }\end{array}$ & Cluster & Side & $x$ & $Y$ & $z$ & Z-score \\
\hline \multicolumn{7}{|c|}{ LM-LMC } \\
\hline IPS & 505 & $\mathrm{R}$ & 22 & -56 & 52 & 4.17 \\
\hline ACG & 60 & & 0 & 14 & 50 & 3.65 \\
\hline LPS & 125 & $\mathrm{R}$ & 42 & -72 & 16 & 4.10 \\
\hline \multicolumn{7}{|c|}{ LB-LBC } \\
\hline IPS & 52 & R & 16 & -54 & 56 & 3.66 \\
\hline FEF & 81 & $\mathrm{R}$ & 24 & -8 & 46 & 3.64 \\
\hline LPS & 85 & $\mathrm{R}$ & 34 & -78 & 20 & 3.44 \\
\hline
\end{tabular}

LM: landmark task, LMC: landmark control, $L B$ : line bisection task, $L B C$ : line bisection control, IPS: intra-parietal sulcus, ACG: anterior cingulate girus, FEF: frontal eye field, LPS: lateral peristriate cortex. 


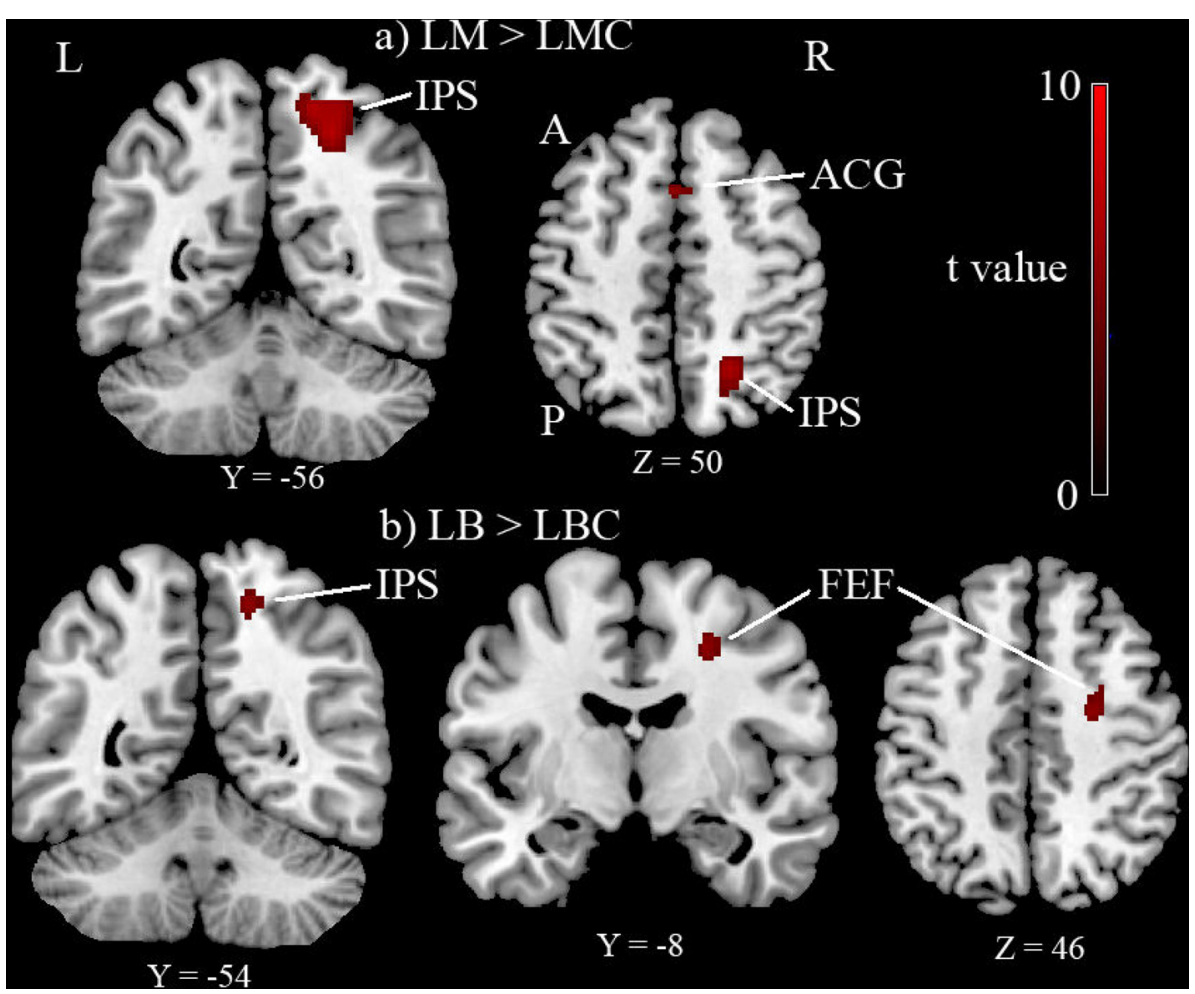

FIGURE 2 | Group results $(n=11)$ thresholded at $p<0.05$ corrected for multiple comparisons. (A) Landmark task (LM) versus Landmark control (LMC) activated intra-parietal sulcus (IPS), anterior cingulate girus (ACG) and lateral peristriate cortex (not shown in this picture). (B) Line bisection task (LB) compared to its control (LBC) activated again IPS, FEF also lateral peristriate cortex not shown in this picture. $A$, anterior; $P$, posterior; $L$, left; $R$, right.
Table 2 |The regions activated for both LM > LMC and LB > LBC contrasts.

\begin{tabular}{lrrrrrr}
\hline Brain area & Cluster & Side & $\boldsymbol{X}$ & $\boldsymbol{Y}$ & $\boldsymbol{Z}$ & Z-score \\
\hline IPS* & 393 & $\mathrm{R}$ & 22 & -56 & 52 & 4.17 \\
LPS & 90 & $\mathrm{R}$ & 42 & -72 & 16 & 4.10 \\
\hline
\end{tabular}

LM: landmark task, LMC: landmark control, LB: line bisection task, LBC: line bisection control. IPS: intra-parietal sulcus, LPS: lateral peristriate cortex. ${ }^{*} R O I$ analysis was performed based on this cluster.

The differential activations between the Landmark and the Line Bisection was assessed with [(LM-LMC) - (LB-LBC)] and $[($ LB-LBC $)-($ LM-LMC $)]$ contrasts. No significant activation passed the corrected threshold ( $p<0.001$ and $\geq 41$ voxels) for these contrasts. Thus, we did not find evidence for any difference in the activation caused by the two tasks.

\section{DISCUSSION}

We used both the Landmark task and the line bisection task to disentangle the question of right hemisphere dominance within the attention networks. The line bisection task is frequently used as a bedside measure of hemi-spatial neglect. Both these tasks require voluntary attention and both activated the dorsal attention system as was predicted. However, the activations were not symmetrical and showed right hemisphere lateralization.

\section{TASK SPECIFIC ACTIVATIONS FOR LANDMARK AND LINE BISECTION}

The present study found right IPS, anterior cingulate girus (ACG) and right LPS activations for the Landmark task (LM-LMC contrast). IPS, ACG and LPS activations (as well as frontal and cerebellar activations) were first reported for the Landmark task by Fink and his coworkers (Fink et al., 2000a, 2001, 2002; Weiss et al., 2003). They reported extensive inferior parietal lobe (IPL) and prefrontal activations during the Landmark task contrary to our findings. This could be due in part to the presentation style of the stimuli and to the choice of a control condition. In most of the above cited work Fink and colleagues presented the stimuli in the four screen quadrants (Fink et al., 2000a, 2002; Weiss et al., 2003) while we did that in the center of the screen. In addition the line stimuli spanned $16^{\circ}$ to $24^{\circ}$ visual angle (greater than our stimuli spanning $6.3^{\circ}$ to $8.6^{\circ}$ visual angle) in the majority of the previous work (Fink et al., 2000a, 2001, 2002). The choice of control condition was simpler in some previous work - to judge if there is a mark on the line or not - (Fink et al., 2000a, 2001). Moreover, in one experiment only a low level baseline was used and highly significant prefrontal and IPL activations were reported (Fink et al., 2002). This suggests that some of the activations reported may not be specific to the Landmark task.

The Line bisection task (LB-LBC contrast) activated IPS, FEF and LPS. This is consistent with the findings of Weiss and his coworkers who required subjects to use a laser pointer to bisect the lines presented on the screen during PET measurements (Weiss et al., 2000, 2003). Although their results well match with our line 


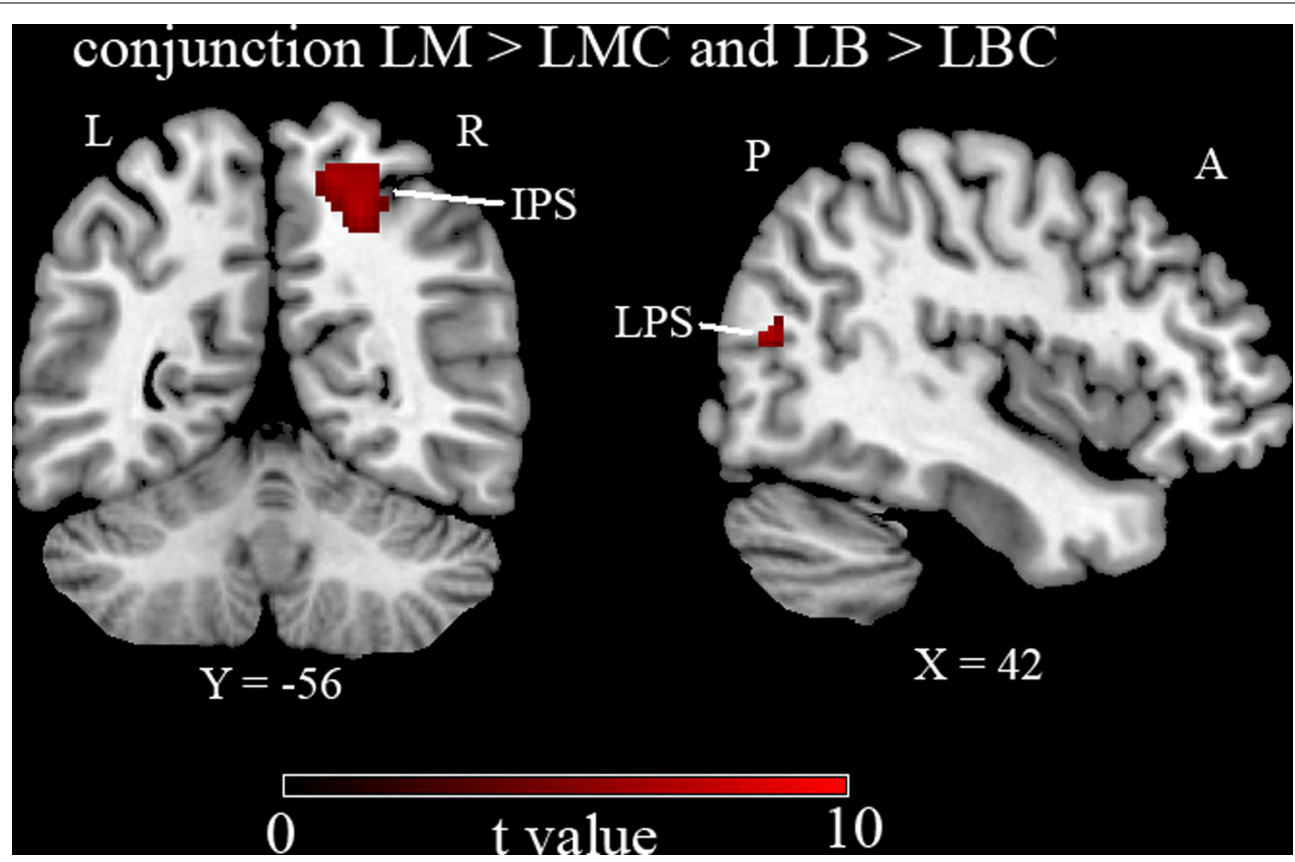

FIGURE 3 | Group results thresholded at $\boldsymbol{p}<\mathbf{0 . 0 5}$ corrected for multiple comparisons. Intra-parietal sulcus (IPS) and lateral peristriate cortex (LPS) activated (please see Table $\mathbf{2}$ for coordinates) commonly for both Landmark and Line Bisection tasks (LM-LMC inclusively masked with LB-LBC). A, anterior; P, posterior; $\mathrm{L}$, left; $\mathrm{R}$, right.

bisection task activations, they reported IPL activity consistently in addition to the activation locations revealed in our results. Again the presentation style of the stimuli could be the reason for the differences in their results. Weiss and coworkers presented the lines in the four screen quadrants though their lines spanned $8.5^{\circ}$ to $9^{\circ}$ visual angle-more similar to ours. Lastly the control condition requiring just pointing to a dot on the screen (Weiss et al., 2000) might not involve some of the spatial processes compared to our line bisection control which requires moving a cursor on a whole line. While the FEF activity in Line bisection (and not in the Landmark task) could be ascribed to increased eye movements required in this task, in fact there were nominally more eye movements in the Line Bisection control condition than in the Line Bisection condition itself (see Section "Behavioral and Eye Movement Data").

In both the Landmark and Line Bisection task conditions subjects should see the line as a whole and then judge the midline. The perceptual judgment of total line length and the estimation of the midline may require a global distribution of the spatial attention to the visual field which activates IPS (Çiçek et al., 2007 ; Molenberghs et al., 2008). The space that must be covered during Landmark and Line Bisection task is more extensive than in the control conditions. In the Line Bisection control condition subjects only move the cursor to the edge of the line and in the Landmark control a very simple visual discrimination is needed. Thus control conditions seem to engage more focal spatial attention.

\section{CONJUNCTION OF LANDMARK AND LINE BISECTION}

The common activations for the Landmark and Line Bisection paradigms were found in the right IPS and right LPS. These findings are also in line with previous functional brain imaging results using line bisection or Landmark paradigms in separate studies (Fink et al., 2001, 2002; Weiss et al., 2000, 2003). IPS, FEF and peristriate regions were activated previously during studies using well controlled spatial attention paradigms with left and rightward cueing (Corbetta and Shulman, 2002; Corbetta et al., 2000; Gitelman et al., 1999; Hopfinger et al., 2000). The common activation regions for these tasks and our tasks overlap with the proposed dorsal voluntary attention network around IPS, FEF and lateral occipital cortex (Corbetta and Shulman, 2002; Corbetta et al., 2000). Although conjunction analysis did not reveal FEF activation this area was active during the Line Bisection task (compared to control).

\section{RIGHT LATERALIZATION}

In the present study, task specific activities for the Landmark and Line bisection tasks as well as conjunction activities were more extensive in the right hemisphere than the left hemisphere. ROI analysis performed based on IPS cluster showed significantly more activation on the right hemisphere for these tasks.

Right hemisphere dominance in line bisection is supported by a combination of electrical and hemodynamic neuroimaging data, as well as lesion data. Patients with right hemisphere lesions show lower performance on line bisection tasks compared to the left hemisphere lesioned groups (Mennemeier et al., 1997). Vallar et al. (1999) reported parietal occipital and frontal fMRI activations during a modified form of line bisection task during which $89 \%$ of the activated voxels were on the right hemisphere. Greater activations on the right hemisphere relative to the left hemisphere during line bisection tasks where shown in other imaging studies using fMRI 
and SPECT (Fink et al., 2000a,b, 2001, 2002; Marshall et al., 1997) and also using transcranial doppler sonography (Flöel et al., 2001, 2002). High-density event-related potential recording during line bisection performance showed early right TPJ and later right parietal (in the vicinity of IPS) localized potentials (Foxe et al., 2003).

Our asymmetrical activations of the dorsal attention network are inconsistent however with the symmetrical activation pattern reported by Corbetta for this network (Corbetta et al., 2000). Corbetta and his coworkers used the Posner paradigm and dissociated cue related activity during which subjects endogenously attended to the left or the right hemi-space from target related activity which required subjects to respond to a stimulus presented from left or right side of the screen. The post-cue period, representing voluntary attention processes, activated the dorsal attention network bilaterally whereas target period engaging involuntary attention processes activated the ventral attention network with right hemisphere dominance. In the present study, the Landmark and Line bisection activated areas overlapping the dorsal attention network more than the ventral parts, possibly because the role of sustained attention is larger than reorienting responses in this task. Nevertheless, the activation of the dorsal attention network was clearly right lateralized. A possible explanation for the more right lateralized activity may lie in the fact that our task required spatial judgments in addition to sustained attention (Kukolja et al., 2006 ). Object based spatial processing requirements (rather than egocentric) in our tasks may be another reason for right lateralization (Galati et al., 2000). Anderson (1996) proposed a mathematical model of line bisection behavior using object based frame of reference and showed simulation results

\section{REFERENCES}

Anderson, B. (1996). A mathematical model of line bisection behaviour in neglect. Brain 119, 841-850.

Bowers, D., and Heilman, K. M. (1980). Pseudoneglect effects of hemispace on a tactile line bisection task. Neuropsychologia 18, 491-498.

Bradshaw, J.L., Bradshaw, J.A., Nathan, G., Nettleton, N. C., and Wilson, L. E., (1986). Leftward error in bisecting the gap between two points: stimulus quality and hand effects. Neuropsychologia 24, 849-855.

Çiçek, M., Gitelman, D., Hurley, R. S. E., Nobre, A., and Mesulam, M. (2007). Anatomical physiology of extinction. Cereb. Cortex 17, 2892-2898.

Corbetta, M., Kincade, J.M., Ollinger,J. M., McAvoy, M. P., and Shulman, G. L. (2000). Voluntary orienting is dissociated from target detection in human posterior parietal cortex. Nat. Neurosci. 3, 292-297.

Corbetta, M., Kincade, J. M., and Shulman, G. L. (2002). Neural systems for visual orienting and their relationships to spatial working memory. $J$. Cogn. Neurosci. 14, 508-523.

Corbetta, M., and Shulman, G. L. (2002). Control of goal-directed and stimulusdriven attention in the brain. Nat. Rev. Neurosci. 3, 201-215.
Fink, G. R., Marshall, J. C., Gurd, J., Weiss, P. H., and Zilles, K. (2001). The neural basis of vertical and horizontal line bisection judgments: an fMRI study of normal volunteers. Neuroimage 14, S59-S67.

Fink, G. R., Marshall, J. C., Shah, N. J., Weiss, P. H., Halligan, P. W., GrosseRuyken, M., Ziemons, K., Zilles, K., and Freund, H.-J. (2000a). Line bisection judgments implicate right parietal cortex and cerebellum as assessed by fMRI. Neurology 54, 1324-1331.

Fink, G. R., Marshall, C., Weiss, H., Shah, N. J., Toni, I., Halligan, P. W., and Zilles, K. (2000b). "Where" depends on "what": a differential functional anatomy for position discrimination in one- versus twodimensions. Neuropsychologia 38, 1741-1748.

Fink, G. R., Marshall, J. C., Weiss, P. H., Toni, I., and Zilles, K. (2002). Task instructions influence the cognitive strategies involved in line bisection judgments: evidence from modulated neural mechanisms revealed by fMRI. Neuropsychologia 40, 119-130.

Fiorelli, M., Blin, J., Bakchine, S., Laplane, D., and Baron, J. C. (1991). PET studies of cortical diaschisis in patients with motor hemi-neglect. J. Neurol. Sci. 104, 135-142.

reminiscent of human subjects' performance. The model proposed that "subjects bisect lines at the point where they perceive the salience of the two line segments created by their bisection mark to be equal". In line with our brain activity results Anderson's model assumed that right hemisphere established most of the magnitude of a point's salience in horizontal axis (Anderson, 1996).

\section{CONCLUSIONS}

Using the Landmark and Line bisection tasks together and looking at the conjunction results might give a clearer view about the brain areas related to the deployment of visuospatial attention. Our data supports the tasks engagement of dorsal fronto-parietal network revolving around IPS and LPS in both tasks and FEF for the line bisection task. The tasks evoked a pronounced right lateralization in agreement with ERP studies and clinical observations. This suggests that not only the ventral attention system, but also the dorsal, may have a predilection for the right hemisphere with some tasks. The overall pattern of results supports a dominant role of the right hemisphere network in voluntary spatial attention. This asymmetry may underlie the dominant role of right hemisphere lesions in hemi-spatial neglect.

\section{ACKNOWLEDGEMENTS}

The authors would like to thank Marc D'Esposito for his valuable contributions on the presented work's experimental design and to the all Knight Lab members. This work was supported by The Scientific and Technological Research Council of Turkey grant SBAG-AYD-429 to MÇ and NINDS grant NS21135 to RTK.

Flöel, A., Knecht, S., Lohmann, H., Deppe, M., Sommer, J., Dräger, B., Ringelstein, E. B., and Henningsen, H. (2001). Language and spatial attention can lateralize to the same hemisphere in healthy humans. Neurology 57, 1018-1024.

Flöel, A., Lohmann, H., Breitenstein, C., Drager, B., Buyx, A., Henningsen, H., and Knecht, S. (2002). Reproducibility of hemispheric blood flow increases during line bisectioning. Clin. Neurophysiol. 113, 917-924.

Foxe, J. J., McCourt, M. E., and Javitt, D. J. (2003). Right hemisphere control of visuospatial attention: line bisection judgments evaluated with highdensity electrical mapping and source analysis. Neuroimage 19, 710-726.

Friston, K. J., Frith, C. D., Turner, R., and Frackowiak, R. S. J. (1995a). Characterizing evoked hemodynamics with fMRI. Neuroimage 2, 157-165.

Friston, K. J., Ashburner, J., Frith, C. D., Poline, J. B., Heather, J. D., and Frackowiak, R.S.J.(1995b).Spatial registration and normalization of images. Hum. Brain Mapp. 3, 165-189.

Friston, K. J., Holmes, A., Worsley, K. J., Poline, J. B., Frith, C. D., and Frackowiak, R. S. J. (1995c). Statistical parametric maps in functional imaging: A general linear approach. Hum. Brain Mapp. 2, 189-210.

Galati, G., Lobel, E., Vallar, G., Berthoz, A., Pizzamiglio, L., and Bihan, D. L., (2000). The neural basis of egocentric and allocentric coding of space in humans: a functional magnetic resonance study. Exp. Brain Res. 133, 156-164.

Gitelman, D. R. (2002). ILAB: a program for postexperimental eye movement analysis. Behav. Res. Methods Instrum. Comput. 34, 605-612.

Gitelman,D.R., Nobre,A.C., Parrish, T. B., LaBar, K. S., Kim, Y. H., Meyer, J. R., and Mesulam, M.-M. (1999). A largescale distributed network for covert spatial attention: Further anatomical delineation based on stringent behavioral and cognitive controls. Brain 122, 1093-1106.

He, B. J., Snyder, A. Z., Vincent, J. L., Epstein, A., Shulman, G. L., and Corbetta, M. (2007). Breakdown of functional connectivity in frontoparietal networks underlies behavioral deficits in spatial neglect. Neuron 53, 905-918.

Heilman, K. M. (1998). Attentional asymmetries. In Brain Asymmetry, R. J. Davidson and K. Hugdahl, eds (Cambridge, MA, MIT Press), pp. 217-234. 
Hopfinger, J. B., Buonocore, M. H., and Mangun, G. R. (2000). The neural mechanisms of top-down attentional control. Nat. Neurosci. 3, 284-291.

Karnath, H. O., Fruhmann Berger, M., Kuker, W., and Rorden, C. (2004). The anatomy of spatial neglect based on voxelwise statistical analysis: a study of 140 patients. Cereb. Cortex 14, 1164-1172.

Karnath, H. O., Himmelbach, M., and Rorden, C. (2002). The subcortical anatomy of human spatial neglect: putamen, caudate nucleus and pulvinar. Brain 125, 350-360.

Katz, N., Hartman-Maeir, A., Ring, H., and Soroker, N. (1999). Functional disability and rehabilitation outcome in right hemisphere damaged patients with and without unilateral spatial neglect. Arch. Phys. Med. Rehabil. 80, 379-384.

Kukolja, J., Marshall, J. C., and Fink, G. R. (2006).Neuralmechanisms underlying spatial judgments on seen and imagined visual stimuli in the left and right hemifields in men. Neuropsychologia 44, 2846-2860.

Manning, L., Halligan, P. W., and Marshall, J. C. (1990). Individual variations in line bisection: a study of normal subjects with application to the interpretation of visual neglect. Neuropsychologia 28, 647-655.

Marshall, J.C., and Halligan, P.W. (1995). Within- and between-task dissociations in visuo-spatial neglect: a case study. Cortex 31, 367-376.

Marshall, R. S., Lazar, R. M., Van Heertum, R. L., Esser, P. D., Perera, G. M., and Mohr, J. P. (1997). Changes in regional cerebral blood flow related to line bisection discrimination and visual attention using HMPAO-SPECT. Neuroimage 6, 139-144.

McCourt, M. E. (2001). Performance consistency of normal observers in forced-choice tachistoscopic visual line bisection. Neuropsychologia 39, 1065-1076.

McCourt, M. E., and Olafson, C. (1997). Cognitive and perceptual influences on visual line bisection: psychophysiological and chronometric analyses of pseudoneglect. Neuropsychologia 35, 369-380.

Mennemeier, M., Vezey, E., Chatterjee, A., Rapcsak, S. Z., and Heilman, K. M. (1997). Contributions of left and right cerebral hemispheres to line bisection. Neuropsychologia 35, 703-715.

Mesulam,M.M.(1981).A cortical network for directed attention and unilateral neglect. Ann. Neurol. 10, 309-325.

Mesulam, M. M. (1990). Large-scale neurocognitive networks and distributed processing for attention, language, and memory. Ann. Neurol. 28, 597-613.

Molenberghs, P., Gillebert, C. R. Peeters, R., and Vandenberghe, R. (2008). Convergence between lesionsymptom mapping and functional magnetic resonance imaging of spatially selective attention in the intact brain. J. Neurosci. 28, 3359-3373.

Mort, D. J., Malhotra, P., Manna, S. K., Rorden, C., Pambakian, A., Kennard, C., and Husain, M. (2003). The anatomy of visual neglect. Brain 126, 1986-1997.

Nalçacı, E., Çiçek, M., Kalaycıoğlu, C., and Yavuzer, S. (1997). Pseudoneglect of males and females on a spatial shortterm memory task. Percept. Mot. Skills 84, 99-105.

Nobre, A. C., Sebestyen, G. N., Gitelman, D. R., Mesulam, M. M.,
Frackowiak, R. S. J., and Frith, C. D. (1997). Functional localization of the system for visuaspatial attention using positron emission tomography. Brain 120, 515-533.

Parton, A., Malhotra, P., and Husain, M (2004). Hemispatial neglect. J. Neurol. Neurosurg. Psychiatry 75, 13-21.

Posner, M. I. (1995). Attention in cognitive neuroscience: an overview. In Handbook of Cognitive Neuroscience, M. S. Gazzaniga, ed (Cambridge, MA, MIT Press), pp. 615-624.

Posner, M. I., and Petersen, S. E. (1990). The attention system of the human brain. Annu. Rev. Neurosci. 13 25-42.

Scarisbrick, D. J., Tweedy, J. R., and Kuslansky, G. (1987). Hand preference and performance effects on line bisection. Neuropsychologia 5, 695-699.

Sobesky, J., Thiel, A., Ghaemi, M. Hilker, R. H., Rudolf, J., Jacobs, A. H., Herholz, K., and Heiss, W. D. (2005) Crossed cerebellar diaschisis in acute human stroke: a PET study of serial changes and response to supratentorial reperfusion. J. Cereb. Blood Flow Metab. 25, 1685-1691.

Talairach, J., and Tournoux, P. (1988). Coplanar Stereotaxic Atlas of the Human Brain. New York, Thieme Medical Publishers, Inc.

Vallar, G. (2001). Extrapersonal visual unilateral spatial neglect and its neuroanatomy. Neuroimage 14, S52-S58.

Vallar, G., Lobel, E., Galati, G., Berthoz, A. Pizzamiglio, L., and Le Bihan, D. (1999). A fronto-parietal system for computing the egocentric spatial frame of reference in humans. Exp. Brain Res. 124, 281-286.
Vandenberghe, R., Duncan, J., Arnell, K. M.,Bishop,S.J.,Herrod, N. J., Owen, A.M., Minhas, P. S., Dupont, P. Pickard, J. D., and Orban, G.A. (2000) Maintaining and shifting attention within left or right hemifield. Cereb. Cortex 10, 706-713.

Weiss, P. H., Marshall, J. C., Wunderlich, G., Tellmann, L., Halligan, P.W., Freund, H. J.,Zilles, K. and Fink, G. R. (2000). Neural consequences of acting in near versus far space: a physiological basis for clinical dissociations. Brain 123, 2531-2541.

Weiss, P. H., Marshall, J. C., Zilles, K., and Fink, G. R. (2003). Are action and perception in near and far space additive or interactive factors. Neuroimage 18 , 837-846.

Conflict of Interest Statement: The authors declare that the research was conducted in the absence of any commercial or financial relationships that could be construed as a potential conflict of interest.

Received: 10 September 2008; paper pending published: 20 November 2008; accepted: 09 April 2009; published online: 08 May 2009.

Citation: Çiçek M, Deouell LY and Knight RT (2009) Brain activity during landmark and line bisection tasks. Front. Hum. Neurosci. (2009) 3:7. doi: 10.3389/neuro.09.007.2009

Copyright (C) 2009 Çiçek, Deouell and Knight. This is an open-access article subject to an exclusive license agreement between the authors and the Frontiers Research Foundation, which permits unrestricted use, distribution, and reproduction in any medium, provided the original authors and source are credited. 